\title{
Correction osteotomy for bilateral varus knee deformity caused by premature epiphyseal closure induced by hypervitaminosis A: a case report
}

\author{
Masatake Matsuoka ${ }^{1}$, Tomohiro Onodera ${ }^{1 *}$ D, Tokifumi Majima ${ }^{1,2}$, Koji Iwasaki ${ }^{1}$, Daisuke Takahashi ${ }^{1}$, Eiji Kondo ${ }^{1}$ \\ and Norimasa Iwasaki ${ }^{1}$
}

\begin{abstract}
Background: A vitamin A derivative, 13-cis-retinoic acid (isotretinoin), has been administered to treat several types of pediatric cancer and has improved survival rates in patients despite being known to induce premature epiphyseal closure. As the number of patients treated by 13-cis-retinoic acid increases, demands for salvage treatment after systemic retinoid therapy are emerging. However, few studies have described the surgical treatment of this disease.

Case presentation: We report a case with bilateral varus knee deformity due to premature epiphyseal closure that occurred during treatment with isotretinoin for neuroblastoma. The patient was successfully treated with correction osteotomy using a Taylor spatial frame in the right knee joint and femoral closed wedge osteotomy using a locking plate in left knee joint. Histopathological examination of the growth plate showed polar irregularity of chondrocytes and decreased cartilage matrix without apoptosis. In contrast, arthroscopic findings showed an intact joint surface. No recurrence of varus deformity was evident on follow-up at 1 year.
\end{abstract}

Conclusions: To the best of our knowledge, this represents the first report of correction osteotomy for varus knee deformity due to premature epiphyseal closure that occurred during treatment with isotretinoin.

Keywords: Retinoid therapy, Premature epiphyseal closure, 13-cis-retinoic acid, Osteopenia

\section{Background}

The first description of poisoning by vitamin A was described in 1596 and again in 1853 by arctic explorers who became ill after ingesting polar bear liver that had a high content of vitamin A $[1,2]$. In the 1940s, Caffey described overdoses of vitamin $\mathrm{A}$ in seven children who presented with skeletal deformities including pain, swollen limbs and radiographic changes [3]. They had been erroneously taking excessive amounts of vitamin concentrate A and D over long periods as oral supplement given them by their parents.

A vitamin A derivative, 13-cis-retinoic acid (isotretinoin), offers a new potential treatment, as the modulation

\footnotetext{
* Correspondence: tomozou@med.hokudai.ac.jp

${ }^{1}$ Department of Orthopaedic Surgery, Hokkaido University Graduate School

of Medicine, North 15 West 7, Kita-Ku, Sapporo 060-8638, Japan

Full list of author information is available at the end of the article
}

of endogenous retinoids induces cytodifferentiation and apoptosis [4]. This agent has thus been administered to treat several types of pediatric cancer and has improved survival rates in patients despite being known to induce premature epiphyseal closure [5-7].

One serious side effect of 13-cis-retinoic acid is irreversible premature epiphyseal closure in growing children [5]. The frequency of epiphyseal closure associated with systemic retinoid therapy is related to dose, age at exposure, and duration of treatment [8]. As the number of patients treated by 13-cis-retinoic acid increases, demands for salvage treatment after systemic retinoid therapy are emerging.

Recently, we encountered a case of bilateral knee varus deformity due to premature epiphyseal closure that occurred during treatment with isotretinoin for the treatment of neuroblastoma. To the best of our knowledge,

(c) The Author(s). 2019 Open Access This article is distributed under the terms of the Creative Commons Attribution 4.0 International License (http://creativecommons.org/licenses/by/4.0/), which permits unrestricted use, distribution, and reproduction in any medium, provided you give appropriate credit to the original author(s) and the source, provide a link to the Creative Commons license, and indicate if changes were made. The Creative Commons Public Domain Dedication waiver (http://creativecommons.org/publicdomain/zero/1.0/) applies to the data made available in this article, unless otherwise stated. 
this represents the first report of correction osteotomy of varus knee deformity occurring during treatment with isotretinoin.

\section{Case-presentation}

A 10-year-old Japanese girl presented to our hospital with progressive pain in the right knee and varus deformity of bilateral knees. She had been diagnosed at three months old as having neuroblastoma from the right adrenal grand with multiple distant metastasis (bone marrow, liver, lung, thoracic wall and skin). Standardized protocol of the Study Group of Japan (A1 protocol [9], consisting of cyclophosphamide $(1200 \mathrm{mg} /$ $\left.\mathrm{m}^{2}\right)$, vincristine $\left(1.5 \mathrm{mg} / \mathrm{m}^{2}\right)$, tetrahydropyranyl adriamycin (pyrarubicin; $40 \mathrm{mg} / \mathrm{m}^{2}$ ), and cisplatin $\left(90 \mathrm{mg} / \mathrm{m}^{2}\right.$ ), was initiated after diagnosis. However, the chemotherapy was stopped due to multiple organ failure. As the next in line therapy 13-cis-retinoic acid was administrated. Administration of isotretinoin was subsequently initiated. She did not receive any other therapy in the last 10 years after administration of isotretinoin. The patient first complained of vague, transient pain in the right knee at 8 years old however she did not receive an additional check up by an orthopaedic surgeon.
The patient was of normal stature at presentation (height, $146 \mathrm{~cm}$ at $87 \mathrm{th}$ percentile; body weight, $37.6 \mathrm{~kg}$ at 62 th percentile; body mass index, $17.6 \mathrm{~kg} / \mathrm{m}^{2}$ at 62 th percentile). Bilateral knees showed varus deformity, with worse deformity in the right knee than in the left knee (hip-knee-ankle angle [HKA]: right, $-23^{\circ}$; left, $-10^{\circ}$ ). The right knee joint showed a range of motion from $10^{\circ}$ to $140^{\circ}$ of flexion. Physical examination and radiographs showed a limb length discrepancy, with the right lower extremity $0.9 \mathrm{~cm}$ shorter than the left lower extremity (right femur, $38.1 \mathrm{~cm}$; right tibia, $34.7 \mathrm{~cm}$; right lower extremity, $72.8 \mathrm{~cm}$; left femur, $38.3 \mathrm{~cm}$; left tibia, $35.4 \mathrm{~cm}$; left lower extremity, $73.7 \mathrm{~cm}$ ). Radiographs and magnetic resonance imaging of the lower extremities revealed medial physeal arrests in both distal femoral physes and in both proximal tibial physes (Figs. 1a, 2) and additional abnormalities in bending of both femoral necks and shortage of both first metatarsals (Fig. 1b, c). According to image findings, a dysplasia epiphysealis multiples should be considered as differential diagnosis. However, the patient did not have family history for dysplasia epiphysealis and received systemic administration of 13-cisretinoic acid for a long-time period. Considering all of these factors, we concluded that the growth disturbance

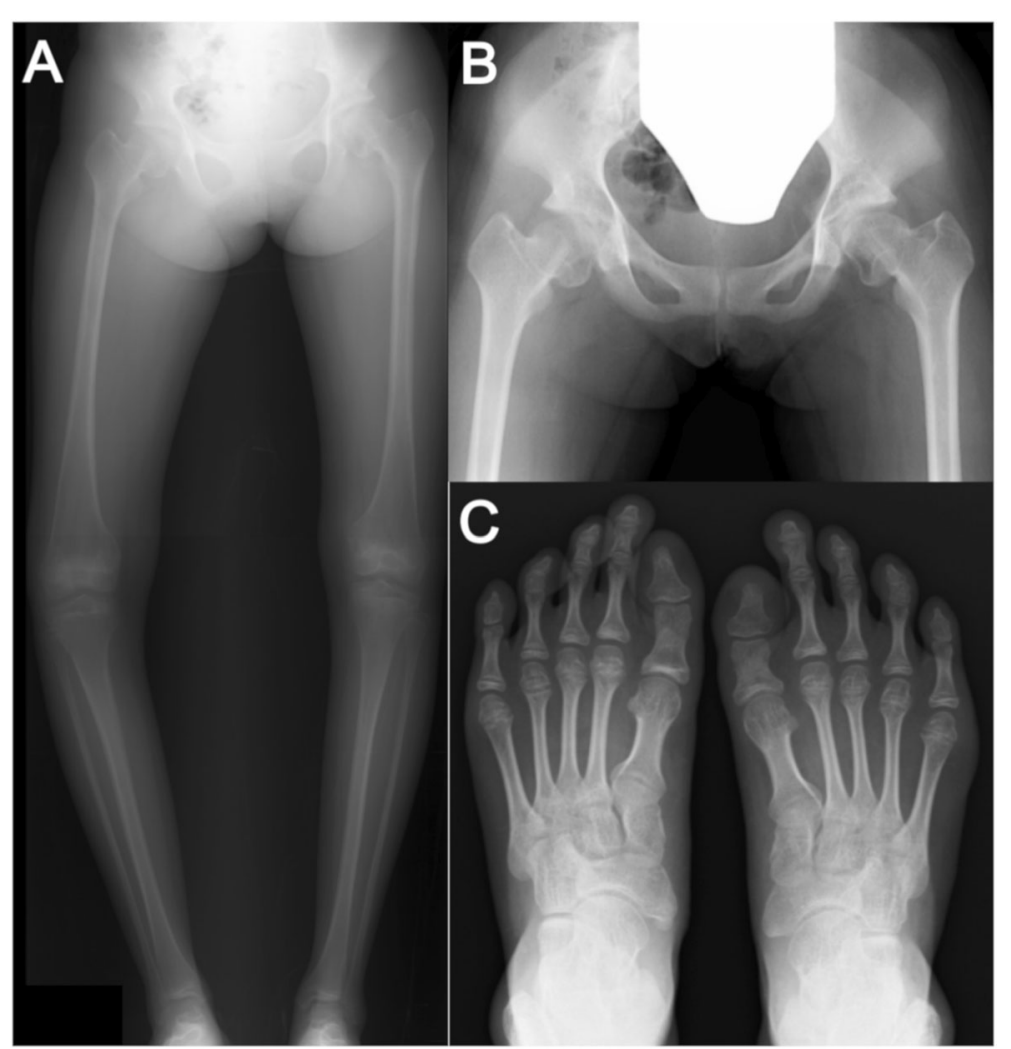

Fig. 1 Abnormalities after long-term treatment for neuroblastoma using isotretinoin, a vitamin A derivative. a Medial physical arrests of both distal femoral physes and distal tibial physes. $\mathbf{b}$ Bending of both femoral necks and deformities of both femoral heads. $\mathbf{c}$ Both first metatarsal bones are short 


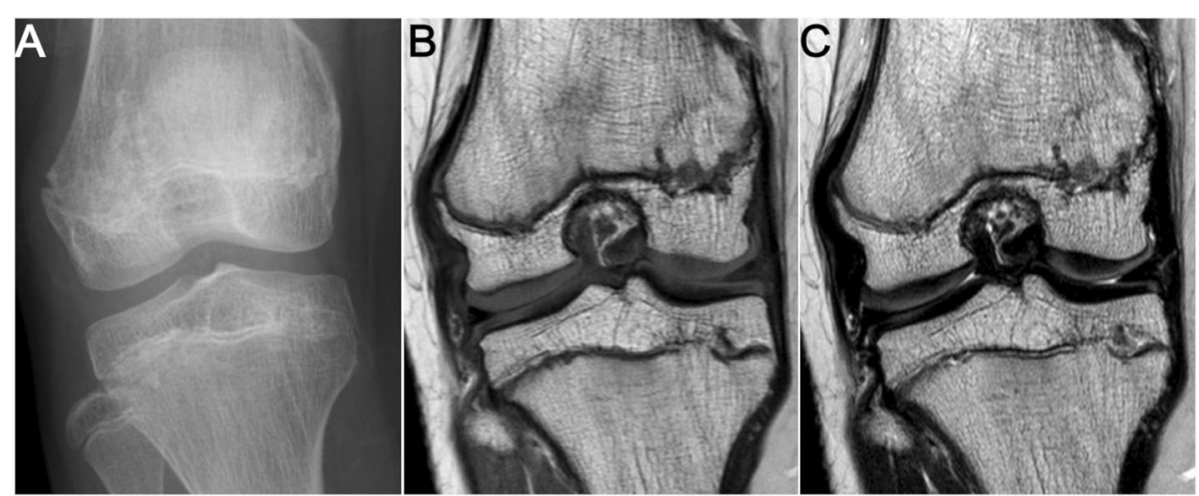

Fig. 2 Close-up view of right distal femoral and proximal tibial physeal arrest. a Anteroposterior radiograph of right knee joint. b, c Coronal T1weighted spin-echo (b) and T2-weighted spin-echo (c) MR images

in this patient was mainly due to premature epiphyseal closure caused by 13-cis-retinoic acid administration.

Weight-bearing anteroposterior (AP) and lateral radiographs were taken preoperatively. The medial proximal tibial angle (MPTA) was $83^{\circ}$ and the mechanical lateral distal femoral angle (mLDFA) was $107^{\circ}$ with $1 \mathrm{~cm}$ leg discrepancy. Accordingly, we performed correction surgery using TSFs to correct both the varus deformity of distal femur and the leg discrepancy. Correction planning was performed using long leg standing AP radiographs. Preoperative planning was carried out using the online Spatial frame software package (http://www.spa tialframe.com). Correction surgery was performed under general anesthesia with the patient supine. First, Taylor Spatial Frames (TSFs) were applied to the distal femur and an Ilizarov frame was fixated to the proximal tibia across the knee joint with tensioned wires and half pins. Second, osteotomy of the distal femur was performed. The angle of the osteotomy was aimed toward perpendicular against the mechanical axis of the lower extremity (Fig. 3a). After mounting the frame on the lower extremity, the mounting parameters were recorded and entered into TSF-dedicated software along with the deformity parameters. All corrections were made gradually after a latency phase of 7 days. The patient was instructed to perform gradual adjustments of the six struts of the TSF three times per day. Correction was continued until HKA had been corrected to $0^{\circ}$ and a 1$\mathrm{cm}$ elongation of the femur, which occurred 18 days after correction. After completing correction, the frame at the tibia was removed and the TSFs were connected with rods (Fig. 3b). Four months later, bone union was confirmed and the external fixator was removed (Fig. 3c). The MPTA was $85^{\circ}$ and MLDFA was $86^{\circ}$ without leg discrepancy (right femur, $38.9 \mathrm{~cm}$; right tibia, $34.7 \mathrm{~cm}$; right lower extremity, $73.9 \mathrm{~cm}$; left femur, $38.4 \mathrm{~cm}$; left tibia, $35.4 \mathrm{~cm}$; left lower extremity, $74.2 \mathrm{~cm}$ ). Arthroscopic investigation revealed that the articular surface of the right knee joint was intact (Fig. 3d). With the consent of the patient and her parents growth plate cartilage was arthroscopically harvested from the non-weight bearing area of the femoral intercondylar fossa to evaluate bone quality affected by 13-cis-retinoic acid administration. We minimized the impact on the growth plate, resulting
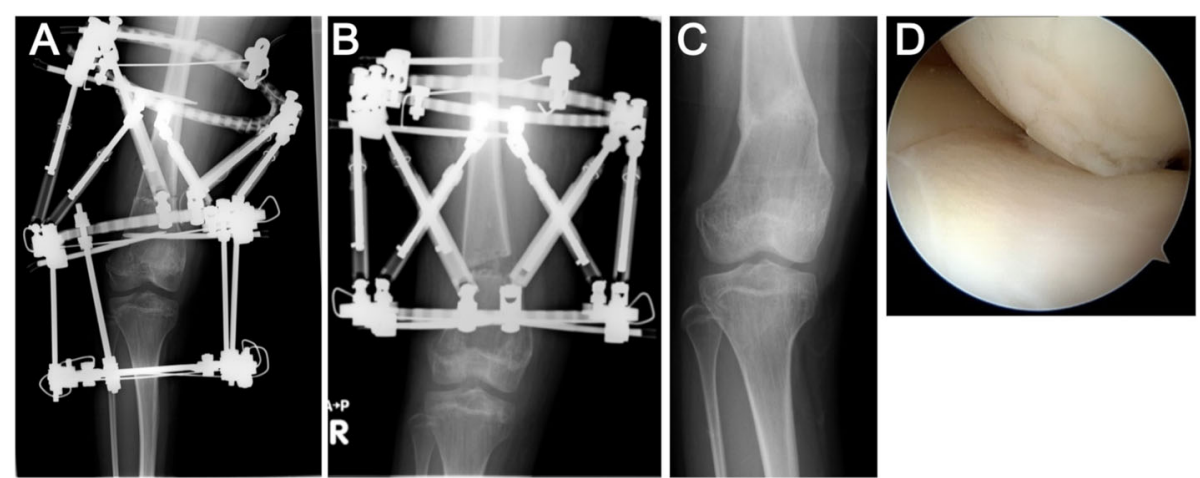

Fig. 3 Postoperative anteroposterior radiographs and arthroscopic images of the patient by TSF. a Immediately after femoral osteotomy. $\mathbf{b}$ Gradual correction was achieved over the course of 18 days. c Four months later, bone union was confirmed and the external fixator was removed. $\mathbf{d}$ Arthroscopic findings showing an intact articular surface 
that no further growth disturbance was observed in her right knee. Specimens $(\$ 2.7 \mathrm{~mm})$ were fixed in $10 \%$ buffered formalin and decalcified in $10 \%$ ethylenediaminetetraacetic acid. Each tissue sample was dehydrated, embedded in paraffin, and sectioned. To investigate chondrocyte apoptosis, the terminal deoxynucleotidyl transferase deoxyuridine triphosphate nick end labeling (TUNEL) assay was performed using an in situ Apoptosis Detection Kit following the instructions of the manufacturer (Takara, Japan). Histological findings revealed that the columnar structure of the growth plate had disappeared and cells were scattered (Fig. 4a). The stainability of Safranin-O staining in growth plate cartilage was dramatically reduced (Fig. 4b). In contrast, cells showing positive results for TUNEL staining were barely detectable (Fig. 4c). Postoperatively, the patient showed no pain in the right knee.

Eight years later, the patient presented again with progressive pain of the left knee joint and planned correction surgery for varus deformity of the left knee (Fig. 5a, b; HKA: $-9^{\circ}$ ). Mild tenderness was evident medially. The left knee joint had a range of motion from $0^{\circ}$ to $145^{\circ}$ flexion. MPTA was $88^{\circ}$ and mLDFA was $103^{\circ}$ without leg discrepancy (right femur, $40.1 \mathrm{~cm}$; right tibia, $35.6 \mathrm{~cm}$; right lower extremity, $75.7 \mathrm{~cm}$; left femur, 40.0 $\mathrm{cm}$; left tibia, $35.8 \mathrm{~cm}$; and left lower extremity, $75.8 \mathrm{~cm}$ ). Accordingly, we performed femoral closed wedge osteotomy because her epiphysis was fused and the center of the varus deformity was located in the distal femur, not in the tibia. Under general anesthesia, a longitudinal surgical incision measuring approximately $10 \mathrm{~cm}$ was made on the lateral aspect of the femur and the iliotibial band was split. The vastus lateralis muscle was then elevated anteriorly and the osteotomy site was exposed. Under fluoroscopic control, a Kirschner wire was inserted to the starting point for distal osteotomy at the lateral femoral epicondyle. Two Kirschner wires were then inserted for an oblique down-sloping wedge. A biplanar osteotomy was then completed and the wedge was gradually closed. A TomoFix Medial Distal Femur (Synthes, Solothurn, Switzerland) for the right knee was inserted on the lateral side of the femur (Fig. 5c). Partial weightbearing was gradually permitted, with full weightbearing permitted from postoperative week 6 . Postoperative MPTA was $88^{\circ}$ and mLDFA was $87^{\circ}$. The inserted plate was removed at 9 months postoperatively. No recurrence of varus deformity was evident on follow-up at 1 year (Fig. $5 \mathrm{~d}$ ). The patient remains able to go about her daily life without experiencing any knee pain.

\section{Discussion and conclusions}

Premature epiphyseal closure caused by hypervitaminosis A can result in various outcomes, from transient abnormalities to permanent deformities. In a study with guinea pigs treated using various doses of vitamin $\mathrm{A}$, the frequency of premature epiphyseal closure depended on the drug dose [10], suggesting that the dose of vitamin A might be important in determining the fate of the epiphyseal plate. In our case, oral isotretinoin was initially administered at $120 \mathrm{mg} /$ day, and was still being administered at $40 \mathrm{mg} /$ day on first admission, with treatment for a total period of 9.8 years from 1 year old. This dose of vitamin A arrested development of the femoral and tibial proximal growth plates, especially on the medial side, resulting in the patient developing severe knee varus deformity by 10 years old.

Retinoic acid, a major metabolite of vitamin A, is known to reduce cartilage matrix synthesis and enhance catabolism of cartilage proteoglycans in vitro [11-13]. These results suggest that the cartilage matrix in the growth plate might undergo histological changes in patients with hypervitaminosis A. In our case, 13-cisretinoic acid accelerated the degradation of cartilage matrix in the growth plate, resulting in early closure of the growth plate. In contrast, apoptosis was not observed in the growth plate cartilage even though 13-cis-

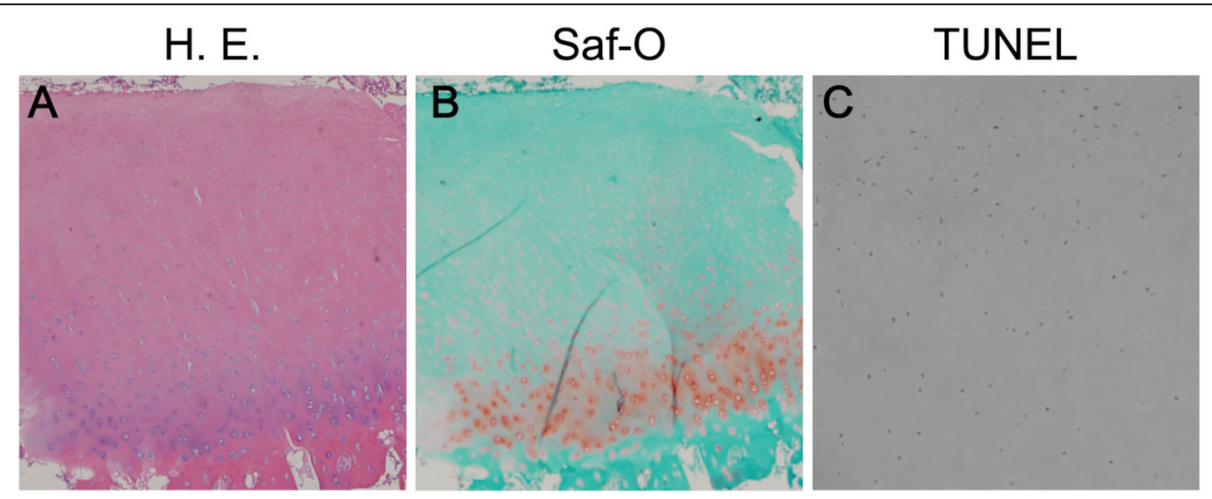

Fig. 4 Histopathological findings of distal femoral growth plate cartilage. a Hematoxylin and eosin staining (HE) shows polar irregularity. b Safranin-O staining (Saf-O) shows decreased cartilage matrix. c TUNEL-stained section shows no obvious apoptosis in the growth plate cartilage. Original magnification: $\times 100$ for $A$ and $B, \times 400$ for $C$ 

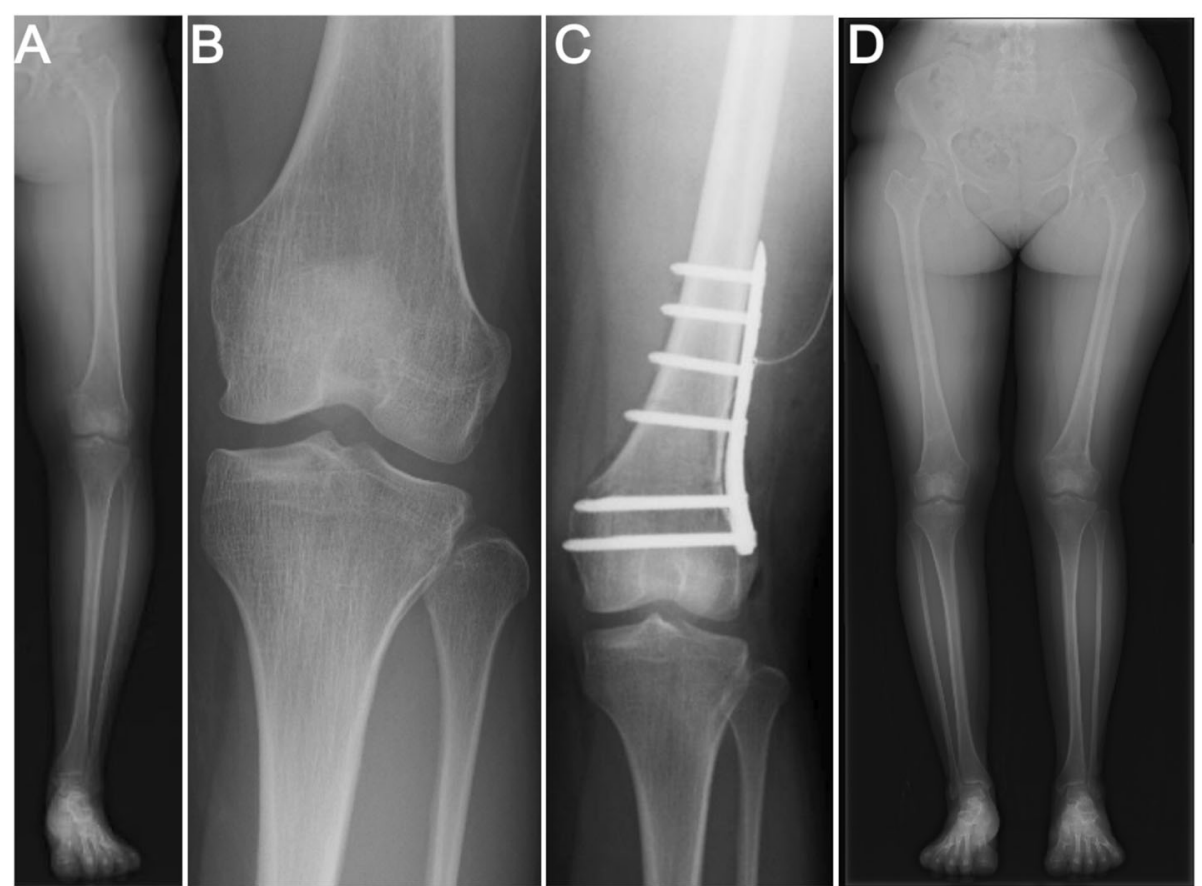

Fig. 5 Pre- and postoperative anteroposterior radiographs of the patient by closed wedge osteotomy. $\mathbf{a}$, $\mathbf{b}$ Left knee joint showing varus deformity. c Immediately after closed wedge osteotomy. d Final follow-up anteroposterior radiographs of the patient

retinoic acid is known to induce cell death in tumors. Additionally, the arthroscopic findings showed that the articular surface was intact. These results suggested that hypervitaminosis A might affect the growth plate cartilage and induce epiphyseal closure without apoptosis.

The complications of osteoporosis and thinning of the long bones is known in hypervitaminosis A [14]. Severe osteoporosis and thinning of the long bones associated with this disease results in consistent occurrence of spontaneous bone fractures. However, few studies have described the surgical treatment of this disease. In our case, the right femoral bone revealed thinning and bone mineral density at L2-L4 did not show severe osteoporosis $\left(0.962 \mathrm{~g} / \mathrm{cm}^{2}\right)$. We decided to extend the external fixation device below the knee only for the period of alignment correction as a safe option, and observed bone union as good as that seen in typical cases. As for the right knee joint, we performed femoral closed wedge osteotomy because her epiphysis was fused and the center of the varus deformity was located in the distal femur, not in the tibia. Bone union was achieved, as in the left knee joint.

This is the first report of arthroscopic and histological findings in premature epiphyseal closure caused by hypervitaminosis A. We believe that the clinician should not hesitate to administrate 13-cis-retinoic acid if the standard chemotherapy is not fully effective for neuroblastoma. In this paper, we reported about a patient who was successfully treated by correction surgery on bilateral premature epiphyseal closure due to systemic administration of 13-cisretinoic acid. Treatment options for growth disturbance are available even after long term administration of 13-cisretinoic acid. In addition, in cases like this a regular check of growth disturbance by a pediatric orthopaedic surgeon is required to insure a long term successful outcome.

\section{Abbreviations}

AP: Anteroposterior; HKA: Hip-knee-ankle angle; mLDFA: Mechanical lateral distal femoral angle; MPTA: Medial proximal tibial angle; TSFs: Taylor Spatial Frames; TUNEL: Terminal deoxynucleotidyl transferase deoxyuridine triphosphate nick end labeling

\section{Acknowledgements}

Special thanks to Yasuhiko Kasahara for his advice and expertise.

\section{Authors' contributions}

TO was involved in the design of the study, performed the clinical assessment, analysis and interpretation of data, and drafted and revised the manuscript. MM, TM, KI and DT were involved in the design of the study, assisted with data interpretation, and revised the manuscript for important intellectual content. EK and NI were involved in the design of the study and the data acquisition and revised the manuscript critically for important intellectual content. All authors have read and approved the final manuscript.

\section{Funding}

No specific funding was received from any funding bodies in the public, commercial or not-for profit to carry out the work described in this article.

\section{Availability of data and materials}

This is a case report of a single patient, to protect privacy and respect confidentiality; none of the raw data has been made available in any public repository. The original operation reports, intra-operative photographs, imaging studies and outpatient clinic records are retained as per normal procedure within the medical records of our institution. All data concerning the case are presented in the manuscript. 


\section{Ethics approval and consent to participate}

Written informed consent was obtained from the patient and her parents for this study.

\section{Consent for publication}

Written informed consent was obtained from the patient and her parents for publication of this case report and any accompanying images.

\section{Competing interests}

The authors declare that they have no competing interests. The authors do not have any commercial or collaborative relationships that could be constructed as biased or inappropriate.

\section{Author details}

'Department of Orthopaedic Surgery, Hokkaido University Graduate School of Medicine, North 15 West 7, Kita-Ku, Sapporo 060-8638, Japan.

${ }^{2}$ Department of Orthopaedic Surgery, Nippon Medical School, 1-1-5,

Senndagi, Bunkyo-ku, Tokyo 113-8603, Japan.

Received: 14 February 2019 Accepted: 4 June 2019

Published online: 15 June 2019

\section{References}

1. Rodahl K, Moore T. The vitamin a content and toxicity of bear and seal liver. Biochem J. 1943;37(2):166-8.

2. Ruby LK, Mital MA. Skeletal deformities following chronic hypervitaminosis A; a case report. J Bone Joint Surg Am. 1974;56(6):1283-7.

3. Caffey J. Chronic poisoning due to excess of vitamin a; description of the clinical and roentgen manifestations in seven infants and young children. Pediatrics. 1950;5(4):672-88.

4. Miller WH Jr. The emerging role of retinoids and retinoic acid metabolism blocking agents in the treatment of cancer. Cancer. 1998;83(8):1471-82.

5. Hobbie WL, Mostoufi SM, Carlson CA, Gruccio D, Ginsberg JP. Prevalence of advanced bone age in a cohort of patients who received cis-retinoic acid for high-risk neuroblastoma. Pediatr Blood Cancer. 2011;56(3):474-6.

6. Nishimura G, Mugishima H, Hirao J, Yamato M. Generalized metaphyseal modification with cone-shaped epiphyses following long-term administration of 13-cis-retinoic acid. Eur J Pediatr. 1997;156(6):432-5.

7. Inamo Y, Suzuki T, Mugishima H. A case of growth failure caused by 13-CISretinoic acid administration after bone marrow transplantation for neuroblasoma. Endocr J. 1999;46(Suppl):S113-5.

8. DiGiovanna JJ. Isotretinoin effects on bone. J Am Acad Dermatol. 2001;45(5): S176-82.

9. Sawaguchi S, Kaneko M, Uchino J, Takeda T, Iwafuchi M, Matsuyama S, Takahashi H, Nakajo T, Hoshi Y, Okabe I, et al. Treatment of advanced neuroblastoma with emphasis on intensive induction chemotherapy. A report from the study Group of Japan. Cancer. 1990;66(9):1879-87.

10. Standeven AM, Davies PJ, Chandraratna RA, Mader DR, Johnson AT, Thomazy VA. Retinoid-induced epiphyseal plate closure in Guinea pigs. Fundam Appl Toxicol. 1996:34(1):91-8.

11. Benya PD, Padilla SR. Modulation of the rabbit chondrocyte phenotype by retinoic acid terminates type II collagen synthesis without inducing type I collagen: the modulated phenotype differs from that produced by subculture. Dev Biol. 1986;1 18(1):296-305.

12. Horton WE, Yamada Y, Hassell JR. Retinoic acid rapidly reduces cartilage matrix synthesis by altering gene transcription in chondrocytes. Dev Biol. 1987;123(2):508-16

13. Morales TI, Roberts AB. The interaction between retinoic acid and the transforming growth factors-beta in calf articular cartilage organ cultures. Arch Biochem Biophys. 1992;293(1):79-84.

14. Barker ME, Blumsohn A. Is vitamin a consumption a risk factor for osteoporotic fracture? Proc Nutr Soc. 2003;62(4):845-50.

\section{Publisher's Note}

Springer Nature remains neutral with regard to jurisdictional claims in published maps and institutional affiliations.

Ready to submit your research? Choose BMC and benefit from:

- fast, convenient online submission

- thorough peer review by experienced researchers in your field

- rapid publication on acceptance

- support for research data, including large and complex data types

- gold Open Access which fosters wider collaboration and increased citations

- maximum visibility for your research: over $100 \mathrm{M}$ website views per year

At $\mathrm{BMC}$, research is always in progress.

Learn more biomedcentral.com/submissions 